\title{
Angiografia Versus Fluxo Fracionado de Reserva na Avaliação do Grau de Importância das Estenoses Coronárias
}

\author{
Fernando Mendes Sant'Anna ${ }^{1,2}$, Expedito Ribeiro da Silva², Leonardo Alves Batista', \\ Fábio Machado Ventura1, Marcelo Bastos Brito', Haroldo Adans Ferraz'1, Leonardo Buczynski', \\ Carlos Alberto Mussel Barrozo ${ }^{1}$, Nico Pijls ${ }^{3}$
}

\begin{abstract}
RESUMO
Introdução: A decisão de realizar uma intervenção coronária percutânea (ICP) em pacientes com angina deve se basear em sinais objetivos de isquemia. A angiografia vem sendo utilizada como padrão-ouro para definição de doença arterial coronariana (DAC), embora suas limitações sejam conhecidas. Por outro lado, o valor da medida do fluxo fracionado de reserva do miocárdio (FFR) na avaliação da DAC está bem estabelecido. Objetivo: Avaliar a acurácia da angiografia em definir as lesões culpadas e sua correlação com o FFR, em todos os pacientes admitidos para ICP eletiva em nossa Instituição, durante determinado período. Método: Foram incluídos no estudo 250 pacientes (471 vasos). Foram avaliadas todas as estenoses $\geq 50 \%$ pela estimativa visual, selecionadas por três cardiologistas independentes, medindo-se o FFR. Quando o FFR era $<0,75$, a lesão foi tratada; se o FFR era $\geq 0,75$, a lesão não foi tratada. Foi realizada QCA off-line de todas as lesões, divididas em moderadas $(<70 \%-327)$ e graves (125). Foram determinados o coeficiente de correlação entre o grau de estenose (\%DE) e o FFR para os dois grupos e a acurácia da estimativa visual da angiografia em definir se uma lesão era ou não isquêmica, tomando-se o FFR como padrão-ouro. Resultados: Foi possível obter o FFR em 96\% das lesões (452). \%DE e FFR médios de $56 \pm 8 \%$ e 0,74 e $76 \pm 6 \%$ e 0,48 para as lesões moderadas e graves. Notouse pobre correlação negativa entre o \%DE e o FFR, principalmente nas lesões moderadas (Spearman $r h o=-0,33, \mathrm{p}<0,0001$ ). A acurácia da estimativa visual da angiografia comparada com o FFR foi de $57 \%$ e $96 \%$, respectivamente, nas lesões moderadas e graves. Conclusões: A angiografia coronária não é um método capaz de prever adequadamente a importância funcional das lesões coronarianas, tornando clara a necessidade de associá-la a um método funcional capaz de orientar corretamente o tratamento dessas estenoses.
\end{abstract}

DESCRITORES: Circulação coronária, fisiologia. Contenedores. Angioplastia transluminal percutânea coronária.

\footnotetext{
Santa Helena Hospital do Coração, Cabo Frio, RJ, Brasil.

2 Instituto do Coração da Universidade de São Paulo, SP, Brasil.

3 Catharina Hospital, Eindhoven, Holanda.

Correspondência: Fernando Mendes Sant'Anna. Rua Safira, 20 -

Portinho - Cabo Frio - RJ - CEP: 28915-400

Telefone: (22) 2647-1984 - Fax: (22) 2644-9262

E-mail: fernandomendes@cardiol.br

Recebido em: 11/03/2007 - Aceito em: 09/04/2007
}

\section{SUMMARY}

Angiography versus Fractional Flow Reserve in the Evaluation of Coronary Stenoses

Introduction: In complex coronary artery disease, it is sometimes difficult to determine which lesions are associated with reversible ischemia. Despite this, the selection of lesions to be stented is often based on the subjectively interpreted angiogram alone. Fractional flow reserve (FFR) is an established objective methodology to indicate which lesions are ischemia-producing. Objective: The aim of this study, in patients admitted for elective percutaneous coronary intervention ( $\mathrm{PCl}$ ), was to evaluate the accuracy of angiography in defining culprit lesions, taking FFR as the gold standard, and also its correlation with FFR. Methods: Two hundred and fifty consecutive patients (471 arteries) scheduled for $\mathrm{PCl}$ were included in this study. All stenoses $\geq 50 \%$ by visual estimation, initially selected to be stented by 3 independent reviewers were assessed by measurement of the FFR. When the FFR was $<0.75$, stenting was performed; when the FFR was $\geq 0.75$, no interventional treatment was given. Offline quantitative coronary angiography (QCA) was performed for all stenoses, divided into intermediate $(<70 \%-327)$ and severe (125). The correlation coefficient between the degree of stenosis (\%DS) and FFR was determined as was the accuracy of the visual assessment of angiography in evaluating ischemia taking FFR as the gold standard. Results: Optimal FFR readings were obtained in 452 lesions (96\%). \%DS and FFR were $56 \pm 8 \%$ and 0.74 for moderate and $76 \pm 6 \%$ and 0.49 for severe stenosis. Concordance between QCA and FFR was poor especially in intermediate stenosis (Spearman's rho $=-0.33, \mathrm{p}<.0001$ ). Visual assessment resulted in accuracies of $57 \%$ and $96 \%$ for intermediate and severe lesions, respectively. Conclusions: Thus, neither visual assessment of an angiogram nor QCA can accurately predict the significance of most intermediate coronary stenoses, stressing the importance of associating these to a functional evaluation of the coronary circulation which can correctly guide treatment of stenosis.

DESCRIPTORS: Coronary circulation, physiology. Stents. Angioplasty, transluminal, percutaneous coronary. 
$S$ abe-se que o fator prognóstico mais importante em pacientes com doença arterial coronariana (DAC) é a presença de isquemia miocárdica ${ }^{1,2}$. Não obstante, muitas intervenções coronarianas percutâneas (ICP) são realizadas baseadas apenas em critérios angiográficos, sem avaliação prévia da existência de isquemia ${ }^{3}$. Ademais, não obstante seus méritos indiscutíveis, são também conhecidas as limitações da angiografia na definição de isquemia miocárdica, especialmente em lesões moderadas ${ }^{4,5}$.

Por outro lado, o valor do fluxo fracionado de reserva do miocárdio (FFR) na definição de isquemia miocárdica já foi amplamente estabelecido ${ }^{6-8}$. Sabe-se que vasos que apresentam $F F R \geq 0,75$ podem ser tratados com segurança de forma conservadora, enquanto um FFR $<0,75$ é sinal de isquemia miocárdica, estando indicada, por vezes, revascularização percutânea ou cirúrgica ${ }^{9-11}$.

Dentro deste contexto, o objetivo desse estudo foi analisar, em pacientes (e lesões) submetidos à intervenção coronária percutânea (ICP), qual a acurácia da estimativa visual da angiografia (EVA) e da angiografia coronária quantitativa (QCA) em apontar quais as lesões responsáveis por isquemia miocárdica, tomando-se o FFR como padrão-ouro.

\section{MÉTODO}

Foram analisados, 250 pacientes encaminhados ao Serviço de Cardiologia Intervencionista do Santa Helena Hospital do Coração para realização de angioplastia coronariana eletiva, no período de outubro de 2004 a abril de 2005. Foram excluídos os pacientes portadores de oclusão crônica do vaso ou doença angiograficamente significativa em tronco da coronária esquerda. Em todos os pacientes, a cineangiocoronariografia diagnóstica havia sido realizada no período entre 2 dias e 3 semanas previamente ao procedimento.

A Figura 1 sintetiza o desenho do estudo. As angiografias de todos os pacientes selecionados para o estudo foram analisadas por três cardiologistas independentes, que desconheciam os sintomas dos pacientes. Assim, as lesões coronarianas foram classificadas em duas categorias, de acordo com a estimativa visual da angiografia: 1) lesões significativas pela EVA, cuja indicação era tratar por meio de angioplastia, com ou sem implante de stent; 2) lesões não significativas pela EVA, cuja indicação era não tratar. Durante a análise das angiografias, em caso de divergência entre os examinadores, a decisão final foi tomada baseada em maioria simples.

\section{Cateterismo cardíaco e medidas pressóricas intracoronarianas}

O cateterismo foi realizado por via femoral, utilizando-se cateteres guia $6 \mathrm{~F}$ ou $7 \mathrm{~F}$ sem furos laterais. Previamente à realização de angiografia, foram administra- das $10.000 \mathrm{Ul}$ de heparina endovenosa e $0,5 \mathrm{mg}$ de nitroglicerina intracoronariana. A seguir, foram realizadas medidas pressóricas intracoronarianas em todos os vasos com estenose $\geq 50 \%$ pela estimativa visual, usandose uma corda-guia 0,014" (PressureWire ${ }^{\circledR} 4$ Sensor, RADI Medical Systems, Uppsala, Suécia), colocada no leito distal de cada coronária a ser analisada, uma de cada vez ${ }^{12}$. Administrava-se adenosina endovenosa através de bainha colocada na veia femoral, na dosagem de $140 \mathrm{mg} / \mathrm{kg} / \mathrm{min}$, para induzir hiperemia máxima. O FFR era determinado automaticamente como a razão entre a pressão média distal da coronária e a pressão média em aorta (medida pelo cateter-guia) durante hiperemia máxima ${ }^{6}$. Todas as estenoses responsáveis por isquemia ( FFR $<0,75$ ) foram tratadas por intervenção percutânea, desde que tecnicamente possível. Estenoses cujo FFR fosse $\geq 0,75$ não foram submetidas à ICP.

\section{Angiografia coronariana quantitativa (QCA) e classificação das lesões}

A angiografia coronariana quantitativa foi realizada off-line, tomando-se a projeção na qual podia-se demonstrar a lesão mais grave, utilizando-se software com algoritmo de detecção automática de contorno (CAAS II, Pie Medical Imaging, Maastricht, The Netherlands), conforme descrito previamente ${ }^{13}$.

As lesões coronarianas foram, então, classificadas em dois grupos: 1) Lesões moderadas: entre 40 e $70 \%$ pela QCA; 2) Lesões graves: $\geq 70 \%$ pela QCA.

\section{Análise Estatística}

Todas as variáveis foram testadas para normalidade pelos testes de Shapiro-Wilks e/ou Kolmogorov-Smirnov. $\mathrm{Na}$ análise descritiva, as variáveis numéricas foram apresentadas como média \pm desvio-padrão (DP) ou medianas e interquartis e as categóricas, como números (n) e porcentagens (\%). O teste do qui-quadrado e o

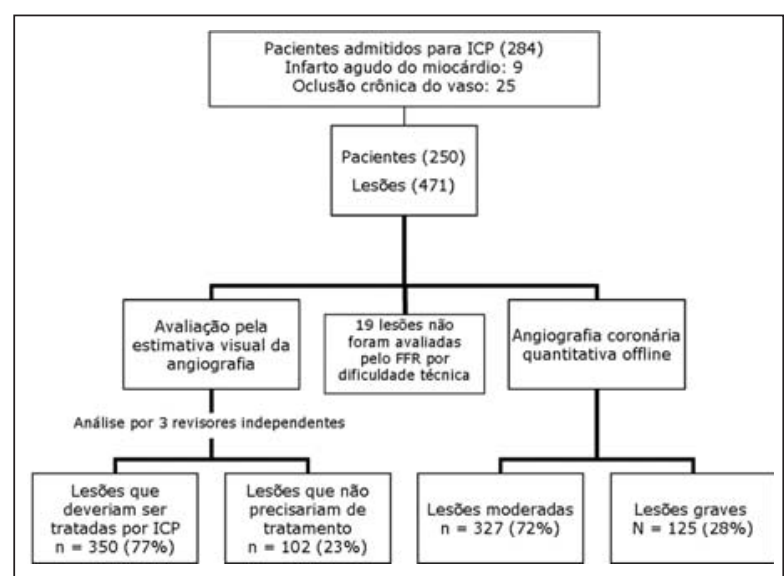

Figura 1 - Visão geral do protocolo de estudo e formação dos grupos. Do terceiro nível em diante os números se referem às lesões, e não aos pacientes. ICP= intervenção coronária percutânea. 
Sant'Anna FM, et al. Angiografia Versus Fluxo Fracionado de Reserva na Avaliação do Grau de Importância das Estenoses Coronárias. Rev Bras Cardiol Invas 2007; 15(2): 119-124.

teste exato de Fisher foram usados para variáveis categóricas; o teste t de Student não-pareado e o teste de Mann-Whitney foram usados para variáveis contínuas. As características angiográficas e o FFR foram comparados entre os grupos de lesões moderadas e graves. O coeficiente de correlação de Spearman (rho) entre a QCA e o FFR foi calculado para ambos os grupos. A sensibilidade, especificidade, valores preditivos positivo e negativo e a acurácia da estimativa visual da angiografia em definir se determinada lesão era responsável por isquemia foram também determinados, tomando-se o FFR como padrão-ouro. A análise estatística foi realizada com o programa Stata SE 9.1 (Stata Corporation, Houston, Texas). Valores de $\mathrm{p}<0,05$ foram considerados estatisticamente significativos, sendo que todos foram bicaudais.

\section{RESULTADOS}

No período de outubro de 2004 a abril de 2005, foram admitidos no Serviço 284 pacientes para se submeterem à angioplastia coronariana, com ou sem implante de stent, sendo excluídos 34 deles, nove por apresentarem infarto agudo do miocárdio (IAM) e 25, oclusão crônica do vaso culpado. Assim, foram incluídos no estudo 250 pacientes, os quais preenchiam os critérios de inclusão.

A Tabela 1 sumariza as principais características clínicas desses pacientes. A maioria dos pacientes (70\%) apresentava angina estável e doença multiarterial (uniarteriais: $30 \%$; biarteriais: $43 \%$; triarteriais: $27 \%$ ). Após a medida do FFR, houve uma inversão desse quadro, conforme se pode observar na Figura 2.

A medida do FFR foi obtida com sucesso em 452 (96\%) lesões analisadas. Em 19 estenoses, não foi possível medir o FFR devido a problemas técnicos (tortuosidade extrema, insucesso ao cruzar a lesão com a corda-guia ou lesão muito distal). Foi realizada angioplastia com implante de stent em 256 estenoses referentes a 193 pacientes, com 100\% de sucesso no procedimento nesse grupo de lesões.

\section{Estimativa visual da angiografia versus FFR}

Houve concordância completa entre os cardiologistas revisores das angiografias em relação à estratégia de tratamento por lesão em $65 \%$ das estenoses e, em 35\%, houve divergência entre um revisor e os outros dois.

Foi indicada ICP em 350 lesões baseado no critério angiográfico da estimativa visual da lesão. Após a medida do FFR, verificou-se que $30 \%$ dessas estenoses (105) não eram responsáveis por isquemia (FFR $\geq 0,75)$ e nenhum tratamento intervencionista foi realizado. Por outro lado, os cardiologistas revisores consideraram que 100 estenoses não tinham importância funcional, devendo ser mantidas apenas sob acompanhamento e tratamento medicamentoso. A medida do FFR mos- trou que $40 \%$ dessas lesões (41) eram isquêmicas (FFR $<0,75)$ e necessitaram de algum tipo de tratamento intervencionista quando tecnicamente possível.

A capacidade estimativa visual da angiografia em detectar isquemia miocárdica, tomando-se o FFR como padrão-ouro, resultou em boa sensibilidade $(85,7 \%)$, porém baixa especificidade $(36,7 \%)$ e valores preditivos positivo $(70 \%)$ e negativo $(59,8 \%)$. A acurácia da EVA foi de $68 \%$ no grupo de lesões estudadas. Quando se considerou apenas as lesões moderadas, a acurácia

TABELA 1

Características clínicas dos pacientes

\begin{tabular}{lc}
\hline & Pacientes (n=250) \\
\hline Idade & $61,0 \pm 10,4$ \\
Sexo feminino, \% & 37,6 \\
Sintomas [n (\%)] & \\
$\quad$ Angina estável & $57(22,8)$ \\
Isquemia silenciosa & $18(7,2)$ \\
Angina instável & \\
Fatores de risco [n (\%)] & $211(84,4)$ \\
Hipertensão arterial & $120(48,0)$ \\
Dislipidemia & $102(40,8)$ \\
História familiar de DAC & $63(25,2)$ \\
Tabagismo & $57(22,5)$ \\
Diabetes & $121(48,4)$ \\
IAM prévio [n (\%)] & $39(15,6)$ \\
ATC prévia [n (\%)] & $8(3,2)$ \\
CABG prévia [n (\%)] & $59 \pm 16$ \\
Fração de ejeção (FE) \% & $176(70,4)$ \\
Doença multiarterial [n (\%)] & \\
\hline Valores representados por média \pm desvio-padrão; \\
CABG= cirurgia de revascularização do miocárdio; \\
IAM= infarto agudo do miocárdio; ATC= angioplastia \\
transluminal coronariana.
\end{tabular}

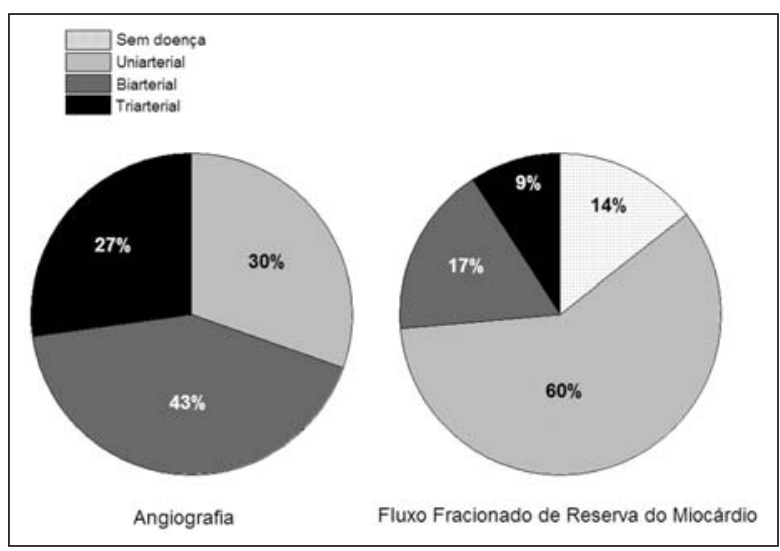

Figura 2 - Extensão da doença coronariana avaliada pela angiografia (A) comparada à extensão funcional da doença segundo o FFR (B). 
Sant'Anna FM, et al. Angiografia Versus Fluxo Fracionado de Reserva na Avaliação do Grau de Importância das Estenoses Coronárias. Rev Bras Cardiol Invas 2007; 15(2): 119-124.

da EVA foi de $57 \%$ versus $96 \%$ nas lesões graves. O erro de interpretação da gravidade da lesão nas lesões moderadas foi em ambas as direções, ou seja, lesões aparentemente graves que se revelaram inocentes e lesões com características angiográficas benignas que se mostraram isquêmicas.

\section{QCA versus FFR}

As características angiográficas das lesões moderadas e graves podem ser observadas na Tabela 2. Observou-se uma correlação inversa entre o grau de estenose pela QCA e o FFR (Figura 3), que foi melhor nas lesões graves $(r h o=-0,55 ; \mathrm{p}<0,0001)$ do que nas moderadas $(r h o=-0,33 ; \mathrm{p}<0,0001)$. No grupo das lesões moderadas, o valor de corte acima do qual mais do que $90 \%$ das lesões eram isquêmicas foi $64 \%$; da mesma maneira, estenoses abaixo de $40 \%$ pela QCA apresentavam possibilidade baixa de serem responsáveis por isquemia (<10\%). Em nosso estudo, o diâmetro luminal mínimo (DLM) pré-ICP não foi um bom parâmetro para se inferir isquemia. Valores de FFR maiores ou menores do que 0,75 ocorreram em todas as faixas de DLM.

\section{DISCUSSÃO}

Os resultados desse estudo confirmam que a angiografia necessita de um complemento para melhor avaliação das estenoses coronarianas, especialmente nas lesões de grau moderado. A QCA pode ser usada como um primeiro passo, ficando o FFR como importante ferramenta reservada para lesões moderadas, especialmente se menores do que $60 \%$ pela QCA.

Considerando-se os consensos do American College of Cardiology/American Heart Association (ACC) AHA) para justificar uma ICP, deve-se provar a existência de isquemia ${ }^{14,15}$. Como em muitos casos os pacien- tes são enviados para ICP sem evidência objetiva de isquemia ${ }^{3}$, e como o FFR é um índice eficaz e específico por lesão para indicar se uma estenose em particular é culpada-9 ${ }^{6-9}$ este estudo sugere que muitos pacientes, especialmente os multiarteriais, são encaminhados para ICP sem seleção adequada das lesões a serem tratadas, especialmente no grupo das lesões moderadas.

A incorporação da informação fisiológica ao arsenal diagnóstico do cardiologista intervencionista pode ser de grande utilidade na tomada de decisões durante ICP, principalmente nas estenoses moderadas, que são as mais encontradas no dia-a-dia. O FFR foi validado tanto em modelos experimentais quanto em estudos em seres humanos, nas mais diversas condições clínicas, nos últimos anos ${ }^{8,16-18}$. É um método simples, reprodutível ${ }^{6,7}$, seguro, além de ser independente do estado

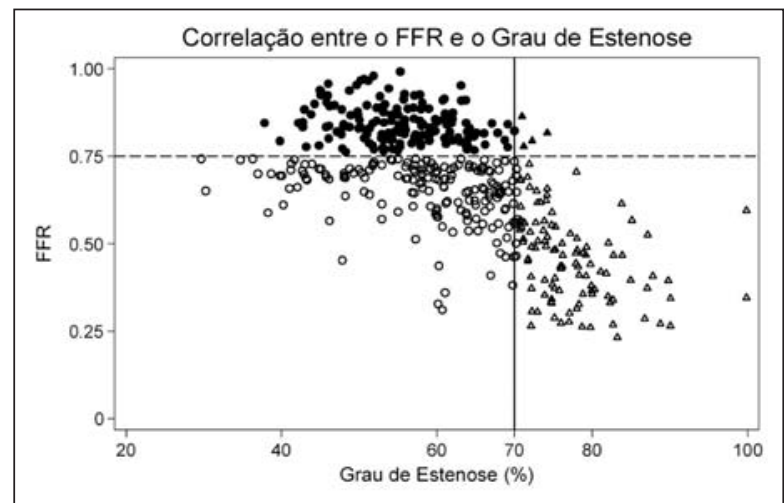

Figura 3 - Correlação entre o grau de estenose medido pela angiografia coronariana quantitativa e o FFR. O círculo corresponde às lesões moderadas e o triângulo às lesões graves. Pode-se notar que, enquanto a maioria das lesões graves apresenta FFR $<0,75$, indicando isquemia, as lesões moderadas se distribuem de forma equivalente acima e abaixo do valor de corte do FFR $(0,75)$.

TABELA 2

Características angiográficas e hemodinâmicas - Análise por lesão

\begin{tabular}{|c|c|c|c|c|}
\hline & \multirow{2}{*}{$\begin{array}{l}\text { Lesões } \\
(\mathrm{n}=452)\end{array}$} & \multicolumn{2}{|c|}{ Grupos de lesões } & \multirow[t]{2}{*}{ Valor de $p$} \\
\hline & & Moderadas $(n=327)$ & Graves $(n=125)$ & \\
\hline Artéria coronária [n (\%)] & & & & 0,34 \\
\hline Descendente anterior & $215(47,6)$ & $162(49,5)$ & $53(42,4)$ & 0,92 \\
\hline Circunflexa & $125(27,6)$ & $89(27,2)$ & $36(28,8)$ & 0,26 \\
\hline Coronária direita & $112(24,8)$ & $76(23,2)$ & $36(28,8)$ & 0,29 \\
\hline ACC/AHA B2 ou C [n (\%)] & $173(38,3)$ & $96(29,3)$ & $77(61,6)$ & $<0,0001$ \\
\hline Diâmetro de referência (mm) & $2,71(2,34 ; 3,02)$ & $2,69(2,34 ; 3,06)$ & $2,72(2,36 ; 2,99)$ & 0,88 \\
\hline Diâmetro luminal mínimo (mm) & $1,02(0,76 ; 1,32)$ & $1,16(0,95 ; 1,4)$ & $0,66(0,54 ; 0,77)$ & $<0,0001$ \\
\hline Grau de estenose (\%) & $62 \pm 12$ & $56 \pm 8$ & $76 \pm 6$ & $<0,0001$ \\
\hline Comprimento da lesão (mm) & $13,6(9,1 ; 19,4)$ & $13,1(8,7 ; 19,0)$ & $14,5(9,8 ; 20,4)$ & 0,06 \\
\hline Fluxo fracionado de reserva & $0,7(0,56 ; 0,81)$ & $0,74(0,68 ; 0,83)$ & $0,48(0,38 ; 0,57)$ & $<0,0001$ \\
\hline
\end{tabular}


hemodinâmico e sofrer pouca influência da microcirculação. Sua correlação com os métodos não-invasivos como a cintilografia do miocárdio, o ecostress com dobutamina e o teste de esforço, é muito boa, apresentando, além disto, a vantagem de ser específico para cada vaso e obstrução ${ }^{8,12}$.

Em contrapartida, tanto a estimativa visual da angiografia quanto a QCA são métodos pouco úteis quando se trata de avaliar lesões moderadas. Fischer et al. ${ }^{4}$ analisaram 83 lesões moderadas (entre $40 \%$ e $70 \%$ ) e compararam a estimativa visual da angiografia e a QCA com o FFR na detecção de lesões isquêmicas. Seus achados sugeriram que tanto a EVA quanto a QCA foram pouco eficazes para predizer o significado da maioria das estenoses moderadas, especialmente aquelas lesões menores do que $60 \%$. Esses dados foram corroborados pelo presente estudo, cujos resultados mostraram que lesões moderadas tanto podem enganar o cardiologista, indicando tratamento quando este não está indicado, quanto deixando de tratar lesões consideradas incorretamente inocentes. Sant'Anna et al. ${ }^{19}$, utilizando o FFR com ferramenta de rotina em todas as angioplastias eletivas durante certo período de tempo, mostraram que $32 \%$ das lesões coronárias e $48 \%$ dos pacientes teriam recebido tratamento incorreto, se somente a estimativa visual da angiografia fosse seguida, enfatizando a utilidade da medida do FFR como ferramenta auxiliar na tomada de decisão durante as intervenções coronarianas percutâneas.

Existem várias limitações no presente estudo: em primeiro lugar, em geral, se considera que o paciente será submetido à cinecoronariografia e depois à ICP eletivas após a realização de algum teste funcional não-invasivo, o que não foi o caso da maioria de nossos pacientes. No entanto, essa é a rotina em muitos serviços no Brasil e em outras partes do mundo $^{3}$ e tentou-se apenas adaptar a essa limitação. Em segundo lugar, o processo de avaliação das lesões coronarianas foi subjetivo, baseado em experiências individuais, ainda que dessa forma reflita com maior precisão o que acontece no mundo real. Ademais, os três revisores eram cardiologistas com vasta experiência no campo da angiografia coronária. Em terceiro lugar, o valor de corte do FFR para definição de isquemia foi de 0,75 , valor este que foi validado baseado em vários estudos $^{6-8}$, porém em populações selecionadas de pacientes uniarteriais, não diabéticos e não revascularizados. A população desse estudo foi mais heterogênea e incluiu pacientes diabéticos, com infarto prévio, alguns já revascularizados e, provavelmente, alguns casos de hipertrofia miocárdica. Pode ser que outro valor de corte devesse ser usado nesses pacientes, o que poderia aumentar a acurácia da angiografia na avaliação dos mesmos.

\section{CONCLUSÃO}

Esse estudo mostrou que a angiografia apresenta baixa correlação com o FFR na definição de existência ou não de isquemia miocárdica, além de acurácia reduzida nas lesões moderadas, tornando clara a necessidade de associá-la a um método funcional capaz de orientar corretamente o tratamento dessas estenoses.

\section{REFERÊNCIAS BIBLIOGRÁFICAS}

1. Davies RF, Goldberg AD, Forman S, Pepine CJ, Knatterud GL, Geller N, et al. Asymptomatic Cardiac Ischemia Pilot (ACIP) study two-year follow-up: outcomes of patients randomized to initial strategies of medical therapy versus revascularization. Circulation. 1997;95(8):2037-43.

2. Shaw LJ, Iskandrian AE. Prognostic value of gated myocardial perfusion SPECT. J Nucl Cardiol. 2004;11(2):171-85.

3. Topol EJ, Ellis SG, Cosgrove DM, Bates ER, Muller DW, Schork NJ, et al. Analysis of coronary angioplasty practice in the United States with an insurance-claims data base. Circulation. 1993;87(5):1489-97.

4. Fischer JJ, Samady H, McPherson JA, Sarembock IJ, Powers ER, Gimple LW, et al. Comparison between visual assessment and quantitative angiography versus fractional flow reserve for native coronary narrowings of moderate severity. Am J Cardiol. 2002;90(3):210-5.

5. Bartunek J, Sys SU, Heyndrickx GR, Pijls NH, De Bruyne B. Quantitative coronary angiography in predicting functional significance of stenoses in an unselected patient cohort. J Am Coll Cardiol. 1995;26(2):328-34.

6. Pijls NH, Van Gelder B, Van der Voort P, Peels K, Bracke FA, Bonnier $\mathrm{HJ}$, et al. Fractional flow reserve: a useful index to evaluate the influence of an epicardial coronary stenosis on myocardial blood flow. Circulation. 1995;92(11):3183-93.

7. De Bruyne B, Bartunek J, Sys SU, Heyndrickx GR. Relation between myocardial fractional flow reserve calculated from coronary pressure measurements and exercise-induced myocardial ischemia. Circulation. 1995;92(1):39-46.

8. Pijls NH, De Bruyne B, Peels K, Van der Voort PH, Bonnier $\mathrm{HJ}$, Bartunek J, et al. Measurement of fractional flow reserve to assess the functional severity of coronary-artery stenoses. N Engl J Med. 1996;334(26):1703-8.

9. Bech GJ, De Bruyne B, Pijls NH, de Muinck ED, Hoorntje JC, Escaned J, et al. Fractional flow reserve to determine the appropriateness of angioplasty in moderate coronary stenosis - A randomized trial. Circulation. 2001;103(24):2928-34.

10. Berger A, Botman KJ, MacCarthy PA, Wijns W, Bartunek J, Heyndrickx GR, et al. Long-term clinical outcome after fractional flow reserve-guided percutaneous coronary intervention in patients with multivessel disease. J Am Coll Cardiol. 2005;46(3):438-42.

11. Legalery P, Schiele F, Seronde MF, Meneveau N, Wei H, Didier K, et al. One-year outcome of patients submitted to routine fractional flow reserve assessment to determine the need for angioplasty. Eur Heart J. 2005;26(24):2623-9.

12. Pijls NH. Optimum guidance of complex $\mathrm{PCl}$ by coronary pressure measurement. Heart. 2004;90(9):1085-93.

13. Reiber JH, Serruys PW, Kooijman CJ, Wijns W, Slager CJ, Gerbrands JJ, et al. Assessment of short-, medium-, and long-term variations in arterial dimensions from computerassisted quantitation of coronary cineangiograms. Circulation. $1985 ; 71(2): 280-8$.

14. Smith SC Jr, Feldman TE, Hirshfeld JW Jr, Jacobs AK, Kern MJ, King SB 3rd, et al. American College of Cardiology/ American Heart Association Task Force on Practice Guidelines; ACC/AHA/SCAI Writing Committee to Update 2001 Guidelines for Percutaneous Coronary Intervention. ACC/ 
AHA/SCAI 2005 guideline update for percutaneous coronary intervention: a report of the American College of Cardiology/American Heart Association Task Force on Practice Guidelines (ACC/AHA/SCAI Writing Committee to Update 2001 Guidelines for Percutaneous Coronary Intervention). Circulation. 2006;113(7):e166-286.

15. Silber S, Albertsson P, Aviles FF, Camici PG, Colombo A, Hamm C, et al. Guidelines for percutaneous coronary interventions. The Task Force for Percutaneous Coronary Interventions of the European Society of Cardiology. Task Force for Percutaneous Coronary Interventions of the European Society of Cardiology. Eur Heart J. 2005;26(8):804-47.

16. De Bruyne B, Hersbach F, Pijls NH, Bartunek J, Bech JW, Heyndrickx GR, et al. Abnormal epicardial coronary resistance in patients with diffuse atherosclerosis but "normal" coronary angiography. Circulation. 2001;104(20):2401-6.

17. De Bruyne B, Pijls NH, Bartunek J, Kulecki K, Bech JW, De Winter $\mathrm{H}$, et al. Fractional flow reserve in patients with prior myocardial infarction. Circulation. 2001;104(2):157-62.

18. Chhatriwalla AK, Ragosta M, Powers ER, Sarembock IJ, Gimple LW, Fischer JJ, et al. High left ventricular mass index does not limit the utility of fractional flow reserve for the physiologic assessment of lesion severity. J Invasive Cardiol. 2006;18(11):544-9.

19. Sant'Anna FM, Silva EE, Batista LA, Ventura FM, Barrozo $\mathrm{CA}, \mathrm{Pijls} \mathrm{NH}$. Influence of routine assessment of fractional flow reserve on decision making during coronary interventions. Am J Cardiol. 2007;99(4):504-8. 